\title{
Nucleon-Nucleon Bremsstrahlung emission of massive Axions
}

\author{
M. Giannotti ${ }^{a}$ and F. Nesti ${ }^{b}$ \\ ${ }^{a}$ Sezione INFN di Ferrara, I 44100 FE \\ E-mail: giannotti@fe.infn.it \\ ${ }^{b}$ Dipartimento di Fisica, Università di L'Aquila, I-67010 Coppito, AQ, and \\ INFN, Laboratori Nazionali del Gran Sasso, I-67010 Assergi, AQ, Italy \\ E-mail: fabrizio.nesti@aquila.infn.it
}

\begin{abstract}
We consider the problem of axion production by bremsstrahlung emission in a nuclear medium. The usual assumption of a massless axion is replaced by more general hypotheses, so that we can describe the emission process for axions with mass up to a few $\mathrm{MeV}$. We point out that in certain physical situations the contribution from non-zero mass is non-negligible. In particular, in the mechanism for the production of Gamma Ray Bursts via emission of heavy axions the axion mass $\left(m_{a} \sim 1 \mathrm{MeV}\right)$ is comparable with the temperature of the nuclear medium, and thus can not be disregarded. Looking at our results we find, in fact, a fairly considerable reduction of the axion luminosity in that mechanism.
\end{abstract}




\section{Introduction}

It is largely believed that the Peccei-Quinn (PQ) mechanism [1 must be realized in nature, since it explains the smallness (or absence) of the CP-violating term in the strong sector of the SM. This problem, known as the Strong-CP problem, is solved dynamically: the $\mathrm{CP}$-violating term is driven to zero by the relaxation of a pseudo-scalar field around its Vacuum Expectation Value (VEV). The field who plays this role, named axion, is the main prediction (still unverified) of the theory. Axions emerge as Pseudo-Goldstone Boson (PGB) modes associated with the (mostly) spontaneously broken PQ symmetry $U(1)_{\mathrm{PQ}}$. The PQ or axion decay constant $f_{a}$, which corresponds to the energy scale of the spontaneous symmetry breaking, characterizes almost all the axion properties, on a phenomenological ground [2]. ${ }^{1}$ More specifically the axion mass is given by the relation

$$
m_{a} / \mathrm{eV} \simeq 0.62 \frac{10^{7} \mathrm{GeV}}{f_{a}}
$$

while its interactions with fermions are measured by $g_{i} \sim m_{i} / f_{a}$, where $m_{i}$ represents the fermion mass (e.g. $m_{e}, m_{N}, \ldots$ for electrons, nucleons, etc.).

Since the PQ mechanism does not fix $f_{a}$, the axion phenomenology is largely modeldependent. However the presently allowed range for $f_{a}$ is rather narrow [2]: terrestrial experiments and astrophysical considerations have in fact excluded all the value of $f_{a}$ up to $10^{10} \mathrm{GeV},{ }^{2}$ while cosmological considerations about axion non-thermal production demand the upper bound $f_{a} \lesssim 10^{11}-10^{12} \mathrm{GeV} .^{3}$

The most stringent lower limits on the axion scale $f_{a}$ derive from astrophysics. Indeed, terrestrial experiments exclude values of $f_{a}$ only up to a few $10^{4} \mathrm{GeV} .{ }^{4}$ However, this value of the axion constant demands the upper limit on the axion mass $m_{a} \lesssim 1 \mathrm{keV}$, by virtue of relation (11). Such a light particle should be emitted from stars of all varieties, and should thereby affect stellar evolution. Thus the axion interaction with the stellar matter must be reduced. This explains the strong limit $\sim 10^{10} \mathrm{GeV}$ on the PQ constant, which is then a direct consequence of relation (11). However, this relation is not a prediction of the $\mathrm{PQ}$ mechanism, and so does not necessarily apply to axions. Actually, the only requirement for the axion field from the PQ mechanism is to dynamically cancel the CP-violating part of the QCD Lagrangian, and this is still possible without satisfying relation (11). This would considerably enlarge the parameter space for the axion, as discussed in [8, 9]. It is then plausible that, in the future axion models, relation (11) could be re-considered.

We can, in fact, refer to a specific example: in [9], it is considered an axion with mass $m_{a} \sim 1 \mathrm{MeV}$ and $\mathrm{PQ}$ constant $f_{a} \sim 10^{6} \mathrm{GeV}$, and it is shown that it can still drive the QCD Lagrangian to its CP-conserving minimum. A particle like that can not be excluded by any phenomenological consideration. In particular, for such a massive axion, the lower

\footnotetext{
${ }^{1}$ Strictly speaking, the energy scale of the spontaneous breaking of the PQ symmetry, say $f_{\mathrm{PQ}}$, does not always correspond to the phenomenological scale $f_{a}$. In general $f_{a}=f_{\mathrm{PQ}} / N$, where $N$ stands for the color anomaly of $U(1)_{\mathrm{PQ}}$ current, and the PQ charges are normalized so that each of the standard fermion families contributes as $N=1$. Therefore, in the Weinberg-Wilczek (WW) model [3], we have $N=N_{g}$, where $N_{g}(=3)$ is the number of fermion families. The same holds in the Dine-Fischler-Srednicki-Zhitnitskii (DFSZ) model [4. Other models of the invisible axion, e.g. the hadronic axion [5] or archion [6], generally contain some exotic fermions and so $N \neq N_{g}$.

${ }^{2}$ In the case of the hadronic axion [5], a small window around $f_{a} \sim 10^{6} \mathrm{GeV}$ can be also permitted.

${ }^{3} \mathrm{~A}$ possibility of relaxing the cosmological limit is discussed in [7].

${ }^{4}$ The somewhat stronger limit, $f_{a} \simeq 10^{5} \mathrm{GeV}$, emerges for an axion heavier than two electrons, from the reactor search of $a \rightarrow e^{+} e^{-}$decay.
} 
limit on the PQ constant is just the terrestrial one $\left(f_{a} \sim\right.$ a few $\left.10^{4} \mathrm{GeV}\right)$, since it can not ruin the stellar evolution process.

The phenomenology of this non-standard axion is quite interesting. As proposed in [10] it can be a key ingredient in explaining the production of Gamma Ray Bursts (GRBs). It can be produced during the merging of two compact objects and then, after its decay, efficiently transfer the gravitational energy of the collapsing system into an ultra-relativistic $e^{+} e^{-}$plasma, the fireball, far from the impact place. Also, since it can be produced in the hot core of type II SN, it can decay into $e^{+} e^{-}$before reaching the stellar surface and, by doing so, transfer a huge amount of energy to a distance of about $1000 \mathrm{Km}$ from the stellar core, helping the SN explosion (thermal bomb). On the other hand, because type Ib/c $\mathrm{SN}$ are smaller, some axions are able to leave their surface and then decay into photons, explaining the observed events of weak GRBs related to type I SN.

The possible existence of heavy pseudo-scalar particles motivates the effort to reconsider the most interesting astrophysical axion processes, removing the usual assumption of zero mass. A particularly interesting example is the well studied nucleon-nucleon axion bremsstrahlung process

$$
N N \rightarrow N N a
$$

where $N$ represents a nucleon. This is the most important axion production mechanism in the hot and dense core of a SN $\left(T \sim 30-80 \mathrm{MeV}, \rho \sim(6-10) \times 10^{14} \mathrm{~g} \mathrm{~cm}^{-3}\right)$, and has received much attention in the past years, in particular after the observation of the neutrino signal from SN1987A. In fact, an axion overproduction in the SN core would ruin the temporal structure of this signal, and this analysis sets the most stringent lower bound on the PQ constant. After the pioneering work of Iwamoto, several other papers discussed the subject [11, but always in the hypothesis $m_{a} \sim 0$. This is clearly very well justified for a standard axion in the SN core, though not for the "axion-GRBs" mechanism of ref. [10, where heavy axions are produced in a medium at a temperature comparable with their mass.

In this paper we discuss the axion emission from nucleon-nucleon axion bremsstrahlung process, replacing the usual assumption of a massless axion with more general hypotheses, so that we can describe the emission process for axions with mass up to a few $\mathrm{MeV}$. In addition, we will consider the effect of the pion mass in the propagator of the nucleonnucleon interaction. Thus we extend the previous analysis, and with these more general assumptions, the above process can be studied in a considerably larger set of physical situations. There are, in fact, physical conditions in which the standard results can not be used, while our hypotheses are still valid, as for the limit of very small axion momentum, and the phenomenology of the "axion-GRBs" mechanism that we have described above; this last will be our main reference example throughout the paper. We will revisit it, showing that the use of the standard results led to an overestimation of the axion luminosity, for fixed axion-nucleon coupling, by a factor $3-10$. This result, however, does not spoil the general idea of reference [10].

The paper is organized as follows: in section 2 we briefly review the "axion-GRB" mechanism of reference [10, 9], while in section 3 we describe the emission process in the nucleon-nucleon axion bremsstrahlung for non-negligible axion mass, and give some numerical results; finally, in section 4, we summarize the results and add some comments. Technical points and some generalizations are discussed in the appendix. 


\section{Axion emission and gamma ray bursts.}

In this section, we briefly review the mechanism of reference [10] that considers a heavy, non-standard axion as the key ingredient in the production of Gamma-Ray Bursts (GRBs). Since this will be our main example, we will frequently refer to it throughout the paper as the "axion-GRBs" mechanism.

The most striking feature of GRBs is that an enormous energy, up to $10^{53-54} \mathrm{erg}$, is released in a few seconds, in terms of photons with typical energies of several hundred keV. The time-structure of the prompt emission and the afterglow observations well agree with the fireball model [12] in which the GRBs originate from the $e^{+} e^{-}$plasma that expands at ultrarelativistic velocities, undergoing internal and external shocks. The Lorentz factor of the plasma needs to be very large, $\Gamma \sim 10^{2}$, and this requires a very efficient acceleration mechanism. In particular, the fireball has to be formed in a region of low baryonic density so that the $e^{+} e^{-}$plasma is not contaminated by more massive matter (baryons). Thus the problem remains how to transform efficiently enough the available energy into the powerful GRBs. Due to the low efficiency of the $\nu \bar{\nu} \rightarrow e^{+} e^{-}$reaction, the models invoking it as a source for the GRBs (see, e.g., [13]) have a lot of difficulty in reaching such large photon luminosities.

In ref. 10, a more efficient mechanism was proposed that invokes the $a \rightarrow e^{+} e^{-}$decay, rather than the reaction $\nu \bar{\nu} \rightarrow e^{+} e^{-}$, where $a$ is a heavy $\left(m_{a} \sim \mathrm{MeV}\right)$ pseudoscalar particle. This can be effectively produced inside the accretion disks that form after the merging of two compact objects, like two Neutron Stars (NS) or NS and Black-Hole (BH), and, decaying far from the disk, can efficiently transfer the gravitational energy of the collapsing system into the ultrarelativistic $e^{+} e^{-}$plasma. The advantages of this mechanism, with respect to the $\nu \bar{\nu} \rightarrow e^{+} e^{-}$annihilation, are obvious: first of all, it is 100 percent efficient, since the decaying axions deposit their energy and momentum entirely in the $e^{+} e^{-}$plasma; in addition, the decay can take place in the baryon free regions, at distances of $1000 \mathrm{~km}$ or larger, and so the plasma can reach a Lorentz factor $\Gamma \sim 10^{2}$.

The parameter range needed for this scenario points to an axion-like particle, with a

mass $m_{a}$ of a few $\mathrm{MeV}$, coupling to nucleons $g_{N} \sim 10^{-6}$ and to electrons $g_{e} \sim 10^{-9}$. This range of coupling constants coincides to that of the invisible axion, with the PQ symmetry breaking scale $f_{a} \sim 10^{6} \mathrm{GeV}$. However, for such a value of $f_{a}$, a standard axion would have a mass of a few $\mathrm{eV}$, whereas the needed particle must have a mass of a few $\mathrm{MeV}$, i.e. about a million times larger. Hence, its mass must not be constrained by the standard relation (10). However, this ultramassive axion cannot be excluded by the existing experimental data and astrophysical limits. Moreover, the relevant parameter window is not far from the present experimental possibilities, and it can be tested with the reactor and beam dump experiments in the close future.

A possible candidate for this new particle could be the "failed" standard axion, which reaches order MeVmass via the Planck scale effects. In this case, however, it cannot be considered for solving the strong CP-problem. A more interesting possibility was presented in [9], in which the mass relation (11) is changed by virtue of the axion interaction with a hidden (mirror) sector of particles. In this last case, the resulting particle is still an axion, meaning it still solves the strong-CP problem.

In the hot medium with temperature $T \sim$ a few MeVand density $\rho \sim 10^{10}-10^{12} \mathrm{~g} \mathrm{~cm}^{-3}$, typical of the central zone of the accretion disk, the nucleons are non-degenerate and nonrelativistic $E_{i} \sim m+\mathbf{p}_{i}^{2} / 2 m$. In these conditions, the main emission process is the nucleon- 
nucleon axion bremsstrahlung $N N \rightarrow N N$ a. Even though this process has been extensively studied in the past, it was always related to the emission of standard axions from the SN core, where $m_{a} / T<10^{-10}$. Therefore, the hypothesis $m_{a}=0$ was always assumed. Also, as we will show in the next section, at the high temperatures inside the SN core, the pion mass in the propagator of the nucleon-nucleon interaction can be neglected. On the other hand, this approximation is not justified at the temperature of a few $\mathrm{MeV}$. A careful analysis of the nucleon-axion bremsstrahlung process is given in the next section. We will show that the use of the standard results for the axion emission rate in [9, 10] led to an overestimation of the axion luminosity for fixed axion-nucleon coupling $g_{n}$, by a factor $\sim 3-10$. This result, however, does not spoil the general idea of ref. [10, since the resulting luminosity is still enough for the production of the GRBs.

Observe, in addition, that the maximal luminosity obtainable in the this mechanism remains essentially unchanged, even if the axion and pion mass effects are taken into consideration. We can briefly explain this result, that will be extensively described at the end of the next section: if the axions are not trapped in the disk, the luminosity function increases with $g_{n}$, until the axions start to interact too strongly with the nuclear matter, and their mean free path becomes smaller than the accretion disk size. Therefore the maximal axion luminosity corresponds to a certain value of the axion-nucleon coupling, $g_{n}^{t r}$, while for a larger coupling the axions become trapped in the disk, and their emission rate decreases. If the non-zero mass effects lower the axion luminosity for fixed coupling $g_{n}$, they also increase the value of $g_{n}^{t r}$, and these two effects balance in the resulting maximal luminosity.

\section{Bremsstrahlung emission of heavy axions}

In this section, we study the nucleon-nucleon axion bremsstrahlung process (2) in the onepion-exchange approximation $(\mathrm{OPE}),{ }^{5}$ in which nucleons interact with each other by exchanging one pion. In addition, we will consider non-degenerate nucleons, and will be focused, for simplicity, on the $n n$ bremsstrahlung ( $n=$ neutron), leaving the more general results for the appendix. Wherever it is possible, throughout this section, we will follow the conventions used in [15] and [16. In particular, we will use " $p_{i}$ " and " $a$ " respectively for the nucleons and for the axion four-momentum, and " $\omega$ " for the axion energy.

All the observables we are interested in can be expressed in terms of the differential axion emission rate: ${ }^{6}$

$$
d \mathcal{N}=d \Pi_{a} \int d \Pi\left\{\mathcal{M}^{2}\right\} f_{1} f_{2}(2 \pi)^{4} \delta\left(p_{1}+p_{2}-p_{3}-p_{4}-a\right),
$$

where $d \Pi=\prod d^{3} \mathbf{p}_{i} /\left[(2 \pi)^{3} 2 E_{i}\right]$ is the Lorentz-invariant phase-space volume element for the four nucleons, while $d \Pi_{a}=d^{3} \mathbf{a} /\left[(2 \pi)^{3} 2 \omega_{a}\right]$ refers to the axion. The occupation numbers of the nucleons $f_{i} \equiv f\left(\mathbf{p}_{i}\right)$ are given by the Maxwell-Boltzmann distribution:

$$
f(\mathbf{p})=\frac{N_{B}}{2}\left(\frac{2 \pi}{m T}\right)^{3 / 2} e^{-\mathbf{p}^{2} / 2 m T},
$$

\footnotetext{
${ }^{5}$ The validity of OPE is discussed in ref. 14.

${ }^{6}$ A note on terminology: throughout this paper, the term "axion number emission rate", or simply "axion emission rate" $\mathcal{N}$, indicates the number of axions emitted in the process per unit time and per unit volume, while the "axion energy emission rate" $Q$ refers to the energy emitted per unit time and volume (axion luminosity per unit volume). The latter is also called "axion energy loss rate" in [16 17], "axion emission rate" in [15], and "axion volume emission rate" in [18].
} 
with normalization $2 \int f(\mathbf{p}) d^{3} \mathbf{p} /(2 \pi)^{3}=N_{B}$. The Pauli blocking factors $\left(1-f_{3}\right)\left(1-f_{4}\right)$ have been omitted, since we are considering non-degenerate nucleons. From the above definition, the axion number emission rate $\mathcal{N}$ and the axion energy emission rate $Q$ are respectively:

$$
\mathcal{N}=\int d \mathcal{N}, \quad Q=\int \omega_{a} d \mathcal{N},
$$

so that $Q / \mathcal{N}$ gives the mean energy of the emitted axions.

For massless axions, the matrix elements squared summed over spins is:

$$
\left\{\mathcal{M}_{0}^{2}\right\} \equiv S \sum_{\text {spin }}\left|\mathcal{M}_{0}\right|^{2}=\frac{64 \pi^{2} \alpha_{\pi}^{2}}{3 m^{2}} g_{n}^{2}\left(\frac{|\mathbf{k}|^{4}}{\left(|\mathbf{k}|^{2}+m_{\pi}^{2}\right)^{2}}+\frac{|\mathbf{l}|^{4}}{\left(|\mathbf{l}|^{2}+m_{\pi}^{2}\right)^{2}}+\frac{|\mathbf{k}|^{2}|\mathbf{l}|^{2}-3|\mathbf{k} \cdot \mathbf{l}|^{2}}{\left(|\mathbf{k}|^{2}+m_{\pi}^{2}\right)\left(|\mathbf{l}|^{2}+m_{\pi}^{2}\right)}\right)
$$

where $g_{n} \simeq m / f_{a}, \alpha_{\pi}=\left(2 f m / m_{\pi}\right)^{2} / 4 \pi \sim 15(f \sim 1$ is a phenomenological constant that accounts for the nucleon-pion interaction) and $S$ is the usual symmetry factor: $S=1 / n$ ! for $n$ identical particles in the final state. ${ }^{7}$ The three-momentum transfers in the "direct" and "exchange" diagrams are indicated respectively with $\mathbf{k}=\mathbf{p}_{2}-\mathbf{p}_{4}$ and $\mathbf{l}=\mathbf{p}_{2}-\mathbf{p}_{3}$ (see figure 4 in the appendix). Observe that $\mathbf{k}^{2} \sim 3 m T$ so that $\mathbf{k}^{2} / m_{\pi}^{2} \sim 0.15(T / 1 \mathrm{MeV})$. Certainly, in a medium with $T \sim$ a few $10 \mathrm{MeV}$, like the $\mathrm{SN}$ core, the pion mass can be neglected, ${ }^{8}$ and the expression for the matrix element squared can be considerably simplified:

$$
\left\{\mathcal{M}_{0}^{2}\right\}_{m_{\pi} \rightarrow 0}=\frac{64 \pi^{2} \alpha_{\pi}^{2}}{m^{2}} g_{n}^{2}\left(1-|\hat{\mathbf{k}} \cdot \hat{\mathbf{l}}|^{2}\right),
$$

where $\hat{\mathbf{k}}=\mathbf{k} /|\mathbf{k}|$ and $\hat{\mathbf{l}}=\mathbf{l} /|\mathbf{l}|$. In this case, from the definitions in (15), one gets:

$$
Q_{0}=\frac{32(3-\beta)}{105} \frac{\rho^{2} T^{7 / 2} \alpha_{\pi}^{2}}{\pi^{3 / 2} m^{13 / 2}} g_{n}^{2} \simeq 5.75 \times 10^{42} T_{\mathrm{MeV}}^{7 / 2} \rho_{12}^{2} g_{n}^{2} \mathrm{erg} \mathrm{cm}^{-3} \mathrm{~s}^{-1}
$$

where $\beta=1.31$ accounts for the contribution of $|\hat{\mathbf{k}} \cdot \hat{\mathbf{l}}|^{2}$ (see the appendix), while $T_{\mathrm{MeV}}=$ $T / 1 \mathrm{MeV}$ and $\rho_{12}=\rho /\left(10^{12} \mathrm{~g} \mathrm{~cm}^{-3}\right)$. Analogously,

$$
\mathcal{N}_{0}=\frac{7}{16}\left(\frac{3-\beta^{\prime}}{3-\beta}\right) \frac{Q_{0}}{T}=1.84 \times 10^{48} T_{\mathrm{MeV}}^{5 / 2} \rho_{12}^{2} g_{n}^{2} \mathrm{~cm}^{-3} \mathrm{~s}^{-1},
$$

where $\beta^{\prime}=1.02$ is defined similarly to $\beta$ (see the appendix). Finally

$$
\bar{\omega}_{0}=Q_{0} / \mathcal{N}_{0} \simeq 1.95 T
$$

is the mean energy of the emitted axions, in the limit of negligible pion and axion masses.

If the assumption that the axion is massless is removed, eq. (6) can no longer describe the axion bremsstrahlung. This can be understood by the following argument. If $m_{a} \neq 0$, we can consider the limit of $|\mathbf{a}| \ll \omega_{a}$. In this limit, we expect $\mathcal{M}^{2} \rightarrow 0$. In fact, as any Goldstone mode, axions interact only derivatively and thus the axion-nucleon coupling must vanish for vanishing axion three-momentum a. Instead, $\mathcal{M}_{0}^{2}$ does not depend on a, and so it can not describe the correct behavior in the above limiting situation. The range of validity of the standard result is indeed $\omega_{a} \gg m_{a}$, which means $|\mathbf{a}| \sim \omega_{a}$.

\footnotetext{
${ }^{7}$ The above result, (6), is the same as the result in [15] and [16]. Observe, though, that in [16] $C_{a n}$ is used in place of $g_{n}$, with $g_{n}=\left(2 m / f_{a}\right) C_{a n}$. In addition, it is defined $\alpha_{a}$ such that $g_{n}^{2}=4 \pi \alpha_{a} C_{a n}^{2}$.

${ }^{8}$ We will frequently refer to the "massless pion limit" or "negligible pion mass effects", etc. throughout the paper. With these expressions we will always intend $\mathbf{k}^{2} / m_{\pi}^{2} \ll 1$.
} 

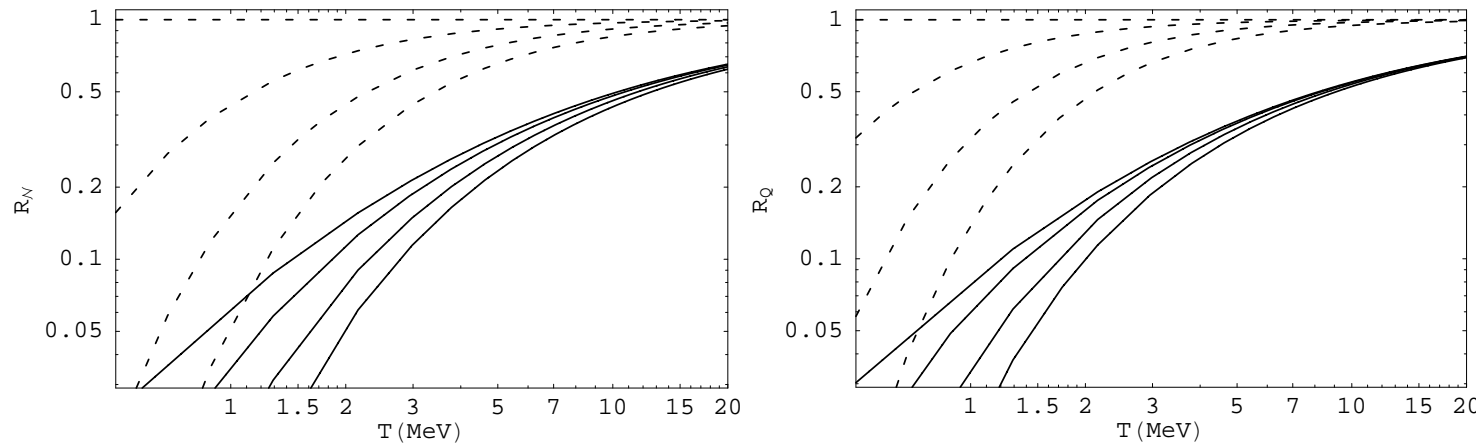

Figure 1: Effects of non-zero axion and pion masses for the axion number emission rate (left panel) and energy emission rate (right panel). Continuous lines represent $R_{\mathcal{N}}\left(m_{a}, m_{\pi}\right)=$ $\mathcal{N}\left(m_{a}, m_{\pi}\right) / \mathcal{N}(0,0)$ (left), and $R_{Q}\left(m_{a}, m_{\pi}\right)=Q\left(m_{a}, m_{\pi}\right) / Q(0,0)$ (right), for different values of the axion mass: $m_{a}=0,1,2,3 \mathrm{MeV}$ from the top to the bottom lines. Dashed lines represent, for the same values of $m_{a}$, the effect of the axion mass alone (i.e. the pion mass is set to zero).

We have, then, calculated the matrix element squared assuming, in place of $m_{a} \sim 0$, the more general hypotheses:

i) The nucleon mass is much larger than both the temperature, $m \gg T$, and the axion mass, $m \gg m_{a}$;

ii) The axion mass is negligible with respect to the momentum transfer $m_{a} \ll \sqrt{m T} \sim$ $30 \mathrm{MeV}\left(T_{\mathrm{MeV}}\right)^{1 / 2}$.

If $T$ is less than a few $10 \mathrm{MeV}$, the above hypotheses are easily satisfied in the range $m_{a} \sim 0$, up to $m_{a} \sim$ a few $T$. Note also that, since the kinetic energy of the emitted axions is $\sim 2-3 T$, hypotheses $(i)$ and $(i i)$ imply that the axion three-momentum is negligible with respect to that of the nucleons.

Assuming the hypotheses above we have found:

$$
\left\{\mathcal{M}^{2}\right\} \equiv S \sum_{\text {spin }}|\mathcal{M}|^{2}=\left(\frac{\mathbf{a}^{2}}{\omega_{a}^{2}}\right)\left\{\mathcal{M}_{0}^{2}\right\} .
$$

Therefore, the more general assumptions above have led to a very simple modification of expression (6). The correction factor $\mathbf{a}^{2} / \omega_{a}^{2}=\left(1-m_{a}^{2} / \omega_{a}^{2}\right)$, which actually is the velocity squared of the emitted axion, becomes fairly irrelevant $(\sim 1)$ in the limit $m_{a} \ll \omega_{a}$. Observe that the result (11) confirms the expected behavior for small axion momentum.

Using the complete matrix element squared (11) we have calculated, numerically, the axion number and energy rates (5). Our results are shown in figure 1, where we plot the effects of pion and axion mass on the emission and the energy emission rates. In the left panel, we show the correction to the axion emission rate

$$
R_{\mathcal{N}}\left(m_{a}, m_{\pi}\right)=\mathcal{N}\left(m_{a}, m_{\pi}\right) / \mathcal{N}(0,0),
$$

where $\mathcal{N}\left(m_{a}, m_{\pi}\right)$ is defined in (15) (see also relation (26) in the appendix), while $\mathcal{N}(0,0)$ is given in (9). We plot this reduction factor for the value of the pion mass $m_{\pi}=135 \mathrm{MeVand}$ for different values of the axion mass, $m_{a}=0,1,2,3 \mathrm{MeV}$ (solid lines). As a comparison, we also show $R_{\mathcal{N}}\left(0, m_{a}\right), m_{a}=0,1,2,3 \mathrm{MeV}$ (dashed lines), where the pion mass is neglected. 
Similarly, in the right panel of figure 1 we consider the axion energy emission rate

$$
R_{Q}\left(m_{a}, m_{\pi}\right)=Q\left(m_{a}, m_{\pi}\right) / Q(0,0)
$$

for $m_{a}=0,1,2,3 \mathrm{MeV}$, with (solid lines) and without (dashed lines) the pion mass contribution. Observe that both $R_{\mathcal{N}}$ and $R_{Q}$ depend on the ratios $m_{a} / T$ and $m_{\pi}^{2} / m T$, and not separately on $m_{a}, m_{\pi}$ and $T$.

As anticipated, in the temperature range of interest for the mechanism [10, $T=2-$ $5 \mathrm{MeV}$, the effect of the pion mass is important, and can be up to one order of magnitude, as expected from the pion propagator suppression. In fact, for $T \lesssim 5 \mathrm{MeV}, m_{\pi}>|\mathbf{k}|$ and $|\mathbf{k}|^{4} /\left(|\mathbf{k}|^{2}+m_{\pi}^{2}\right)^{2}$ can be roughly approximated as $|\mathbf{k}|^{4} / m_{\pi}^{4} \sim 0.1-0.6$, for $T=2-5 \mathrm{MeV} .^{9}$ Besides this, also the axion mass induces a suppression, which is about a factor 2 , for $m_{a} \sim T \sim$ a few MeV. Clearly, when the mass is greater than the temperature, the suppression becomes exponential because of the nucleons Boltzmann distribution.

The ratio $Q / \mathcal{N}$ gives the mean energy $\bar{\omega}_{a}$ of the emitted axions. In figure 2 we present the average kinetic energy divided by the temperature: $\bar{E}_{\text {kin }} / T=\left(\bar{\omega}_{a}-m_{a}\right) / T$. This can be compared with the case of thermal axions, for which $\bar{E}_{\text {kin }} / T$ is either $3 / 2$ (non-relativistic) or 3 (relativistic). The dashed line is calculated, again, neglecting the pion mass. Thus it corresponds to the limit of high temperature. ${ }^{10}$ In this limit, and for massless axions, the dashed line of figure 2 reproduces the result (10) as $\bar{E}_{\text {kin }} / T \simeq 1.95$ (left endpoint). We see that, in this case, the emitted axions are less energetic than thermal axions. At lower temperatures, the pion contribution is important, and the mean kinetic energy per temperature increases. In fact, for massive pions, low energy processes are more difficult, and the axions are emitted only by the most energetic nucleons. For example, at $T=1 \mathrm{MeV}$ and for massless axions, $\bar{E}_{\text {kin }} \simeq 2.48 \mathrm{~T}$, about $30 \%$ more than in the high temperature limit. The dependence of $\bar{E}_{\text {kin }} / T$ on the axion mass is less strong. It changes not more that $5 \%$ for the $m_{a}$ in the range $0-5 T$.

Let us finally discuss the axion mean free path $\lambda$, in the nuclear medium. This is defined as

$$
\lambda^{-1}=\frac{1}{2|\mathbf{a}|} \frac{d \mathcal{N}(-a)}{d \Pi_{a}}=\frac{1}{2|\mathbf{a}|} \int d \Pi\left\{\mathcal{M}^{2}\right\} f_{1} f_{2}(2 \pi)^{4} \delta\left(p_{1}+p_{2}-p_{3}-p_{4}+a\right),
$$

that reduces to the definition (2) in [18] in the limit of zero axion mass. The notation $d \mathcal{N}(-a)$ means that the axion four-momentum must be taken with the opposite sign in the $\delta$ function, with respect to expression (31). In fact, in this case, the relevant process is the axion absorption $N N a \rightarrow N N$, with the axion in the initial state.

Of course $\lambda$ is a function of the axion energy. A simple case, which is particularly interesting for the discussion below, is when the mean free path is large enough for the absorbed axions to be non-thermal. In this case, the average axion energy was calculated above (see figure 21). With this assumption, in the limit of negligible pion and axion mass, $\lambda$ can be well-approximated by the relation (see the appendix):

$$
\lambda_{0}^{-1}=4.2 \times 10^{6} T_{\mathrm{MeV}}^{-1 / 2} \rho_{12}^{2} g_{n}^{2} \mathrm{~cm}^{-1},
$$

where we used $\bar{\omega}_{a}=1.95 \mathrm{~T}$.

\footnotetext{
${ }^{9}$ This effect was estimated approximately in ref. [18, and it is also discussed in ref. [16, both times in the massless axion approximation.

${ }^{10}$ Moreover, since $T$ is high, it describes the situation with $m_{a}$ very large $\left(m_{a}\right.$ up to $\left.5 T\right)$.
} 


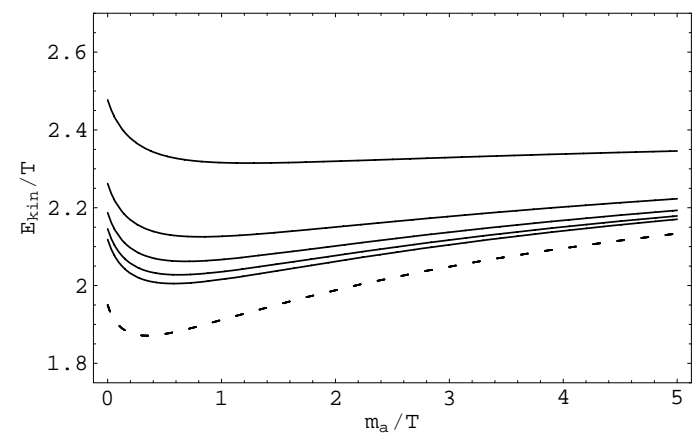

Figure 2: Mean kinetic energy of the emitted axions, divided by the temperature, with (continuous) or without (dashed) the effect of nonzero pion mass, for different values of the temperature: from the top to the bottom solid line $T=1,5,9,13,17 \mathrm{MeV}$. Note that the dashed line, where $m_{\pi}=0$, also represents the very high temperature limit $3 m T \gg m_{\pi}^{2}$.

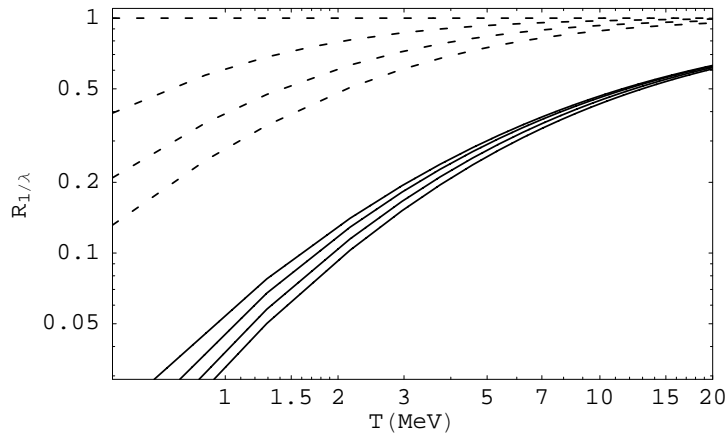

Figure 3: Reduction of the inverse mean free path of the emitted axions, due to non-zero axion and pion masses. Continuous lines represent $R_{1 / \lambda}\left(m_{a}, m_{\pi}\right)=\lambda^{-1}\left(m_{a}, m_{\pi}\right) / \lambda^{-1}(0,0)$ for different values of the axion mass: from the top to the bottom line $m_{a}=0,1,2,3 \mathrm{MeV}$. Dashed lines represent $R_{1 / \lambda}\left(m_{a}, 0\right)$ for the same values of the axion mass.

In figure 3 we have shown the reduction of the inverse mean free path due to finite axion and pion mass effects:

$$
R_{1 / \lambda}\left(m_{a}, m_{\pi}\right)=\lambda^{-1}\left(m_{a}, m_{\pi}\right) / \lambda^{-1}(0,0)
$$

We notice that the pion mass contribution is similar in the absorption and in the emission processes. Thus, at low temperatures, we expect a suppression of (14) by a factor $\sim|k|^{4} / m_{\pi}^{4}$. On the other hand, the axion mass plays a different role in the two processes. In the appendix it will be shown that non-zero axion mass effects can be approximately accounted as

$$
R_{1 / \lambda}\left(m_{a}, 0\right) \simeq 0.6\left(1-m_{a}^{2} / \bar{\omega}_{a}^{2}\right)^{1 / 2} e^{\bar{\omega}_{a} / 2 T} K_{1}\left(\bar{\omega}_{a} / 2 T\right),
$$

where $K_{1}$ is the modified Bessel function of the second kind. For example, for $T=2 \mathrm{MeV}$, $m_{a}=1 \mathrm{MeV}$, we find $R_{1 / \lambda} \simeq 0.8$, in accordance with figure 3 The contribution from the pion mass lowers the above result to $\sim 0.1$ (using $\sim|k|^{4} / m_{\pi}^{4} \sim 0.1$ for $T=2 \mathrm{MeV}$, we would predict $R_{1 / \lambda} \sim 0.08$ ).

We can now understand better what was discussed at the end of the last section about the "maximal luminosity" obtainable in the "axion-GRBs" mechanism of ref. [10]. As explained, in order to have a large luminosity, the axions must be not trapped in the accretion disk. This is the case for $g_{n}<g_{n}^{t r}$, so that the axion mean free path does not exceed the accretion-disk radius. In the limit of negligible pion and axion mass, from (13) we get the relation for $g_{n}^{t r}$ in terms of the accretion disk ratio $R_{100}=R / 100 \mathrm{Km}$, reported in [9, 10]: $g_{n}^{t r} \simeq 1.5 \times 10^{-7} R_{100}^{-1 / 2} \rho_{12}^{-1} T_{\mathrm{MeV}}^{1 / 4}{ }^{11}$ The maximal axion luminosity (per volume) corresponds, roughly, to $Q_{\mathrm{Max}} \equiv Q\left(g_{n}=g_{n}^{\text {tr }}\right)$. We have shown that both the axion and pion mass contribute to reducing the axion luminosity, for fixed axion-nucleon coupling. However, these effects reflect also on the axion mean free path and consequently on $g_{n}^{t r}$, which increases, balancing the reduction of the axion energy emission rate. This is clear comparing figure 3 with figure 1-right. Suppose, for example, that $T=2 \mathrm{MeV}$ and $m_{a}=1$

\footnotetext{
${ }^{11}$ Observe that in references 9] 10 this result was slightly overestimated because of the assumption $\bar{E}_{\text {kin }} \simeq 3 T$.
} 


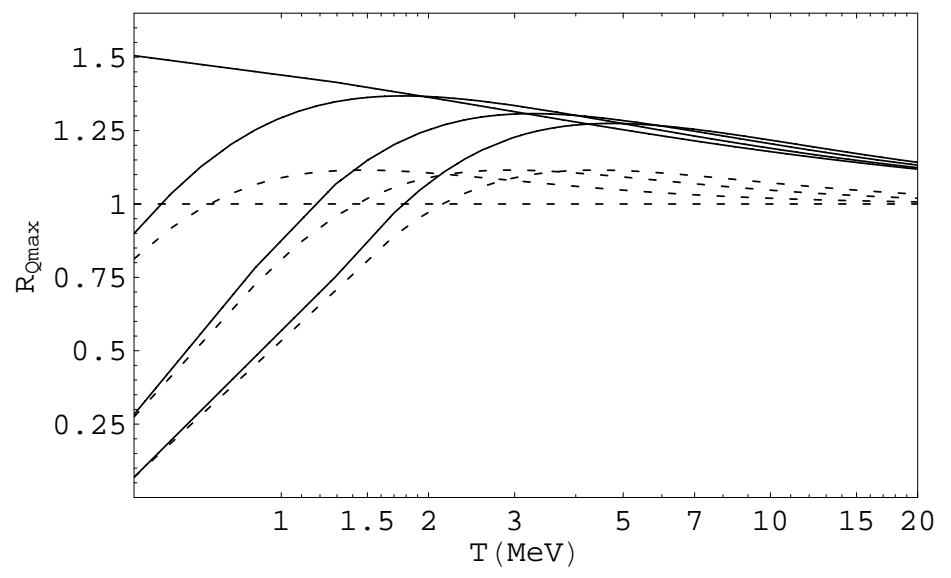

Figure 4: Modification of the maximal GRB luminosity from non-zero axion and pion mass effects: $R_{Q \operatorname{MAX}}=Q_{\operatorname{MAX}}\left(m_{a}, m_{\pi}\right) / Q_{\operatorname{MAX}}(0,0)$. Dashed lines are calculated omitting the effect of nonzero pion mass. The values of the axion mass are $m_{a}=0,1,2,3 \mathrm{MeV}$.

$\mathrm{MeV}$. We find a reduction of the axion luminosity by a factor of 7 , compensated by an increment of the axion mean free path by a factor of about 9 . Thus we find that $Q_{\mathrm{Max}}$ is about 1.25 times larger, in the above conditions. This is confirmed in figure 4 where we have presented the effect of the axion and pion masses on the maximal axion luminosity, as a function of the temperature. As anticipated, there are no significant changes in the resulting maximal luminosity, except for a factor of $\sim 2-4$ in the region of very low temperatures.

\section{Conclusions}

The existence of a heavy non-standard axion with mass $\sim \mathrm{MeV}$ can not be excluded by any phenomenological consideration. Indeed, explicit models have been considered in the past which relax the relation (11) between $f_{a}$ and $m_{a}$ and which allow the axion mass to be very large. Interestingly, a quite efficient mechanism for the GRB production was proposed in [10, which requires a massive axion-like particle to transfer gravitational energy into the $e^{+} e^{-}$fireball. The conditions in which this particle is produced, temperature $T \sim$ a few $\mathrm{MeV}$, and density $\rho \sim 10^{10}-10^{12} \mathrm{~g} \mathrm{~cm}^{-3}$, favor the nucleon-nucleon axion bremsstrahlung production. Even if this process has been studied extensively, the problem of the emission of an axion with mass not negligible with respect to the temperature of the medium has never been considered in the past. Thus, in particular, the luminosity reported in [10] is overestimated by a factor of $3-10$.

In this paper, we have re-studied the problem of nucleon-nucleon axion bremsstrahlung, relaxing some of the old hypotheses in order to enlarge the possibility for applications. In this section, we summarize the most interesting points of this paper:

i) The usual assumption of negligible axion mass has been replaced with more general hypotheses: $a$ ) the nucleon mass is much greater than both the temperature, $m \gg T$, and the axion mass, $\left.m \gg m_{a} ; b\right)$ the axion mass is negligible with respect to the momentum transfer $m_{a} \ll \sqrt{m T} \sim 30\left(T_{\mathrm{MeV}}\right)^{1 / 2} \mathrm{MeV}$. If $T$ is less than a few $10 \mathrm{MeV}$, the above hypotheses are easily satisfied in the range $m_{a} \sim 0$, up to $m_{a} \sim T$ or so. As we have shown, $(a)$ and $(b)$ imply that the axion three-momentum is negligible with respect to that of the nucleons. 
ii) We have computed the axion number and energy emission rate $\mathcal{N}$ and $Q$ in the conditions of point $(i)$. Besides the obvious kinematic suppression due to the reduction of the axion phase space, there is also a less trivial dynamical effect of non-zero axion mass, due to the change (111) of the matrix element squared. We also have considered the effects of finite pion mass, which are important for temperatures below $\sim 6 \mathrm{MeV}$. The final results are presented in figure 10 in which we show the axion number emission rate (left panel) and energy emission rate (right panel), normalized to the standard one $\left(m_{a}=m_{\pi}=0\right)$, both considering (continuous lines) or neglecting (dashed lines) the effects of nonzero pion mass. As we see, in the temperature range of interest for the mechanism [10], 2-5 MeV, the suppression due to $m_{\pi} \neq 0$ is important, and amounts to a factor from $\sim 2$ up to one order of magnitude, as expected from the pion propagator suppression. On the other hand, the axion mass induces a suppression which is around a factor of 2 , for an axion mass of a few $\mathrm{MeV}$, and becomes much larger when the mass exceeds the temperature, mainly because of the Boltzmann exponential suppression.

iii) The two effects of non-negligible axion and pion mass show that the result of reference [10] overestimates the luminosity for fixed axion-nucleon coupling by roughly an order of magnitude.

iv) The reduction of the nucleon-axion interaction rate induces an increment of the axion mean free path in the medium. Our numerical results are presented in figure 3 where we show the behavior of $R_{1 / \lambda}\left(m_{a}, m_{\pi}\right)=\lambda^{-1}\left(m_{a}, m_{\pi}\right) / \lambda^{-1}(0,0)$, with $\lambda\left(m_{a}, m_{\pi}\right)$ the mean free path for fixed axion and pion mass. Again, for the dashed lines the contribution of finite pion mass was neglected.

$v$ ) Even if the actual luminosity for fixed axion-nucleon coupling is reduced by almost an order of magnitude, the maximal luminosity obtainable in the "axion-GRBs" mechanism of ref. [10] does not considerably change. In fact, the reduction of the axion emission rate is compensated by the increasing of the mean-free path which ultimately allows a larger value for the axion-nucleon coupling constant, without trapping the axions in the disk. We note however that for this to happen one should be able to take a larger axion-nucleon coupling, which is usually not easy, since $g_{n} \sim m / f_{a}$ is only slightly model dependent.

vi) We notice, to conclude, that our corrections to the results of ref. 9, 10, even if nonnegligible, do not spoil the general idea of the "axion-GRBs" mechanism. As remarked in ref. [10, the emitted axions can still produce the GRBs, even if their luminosity is reduced by one order of magnitude.

We also mention that for an axion such as the one described in [10] and [8, 9, the brems strahlung process inside the SN core is very well described by the standard formula (8). In particular, the effect of axion and pion masses on the limit on the axion-nucleon coupling, reported in ref. [8, 9, is negligible.

\section{Acknowledgments}

We are grateful to Z. Berezhiani and Denis Comelli for very useful discussions and suggestions. We also thank A. Drago for many explanations about the mechanism of GRBs production via emission of axion-like particles. We are finally thankful to Elizabeth Price for the careful reading of this paper. 


\section{Appendix}

Here we discuss in detail some technical points necessary for the analysis of the nucleonnucleon axion bremsstrahlung process.

First of all, we consider closely the matrix element squared for the process $N N \rightarrow$ $N N$ a, for a massive axion, assuming the One Pion Exchange approximation (OPE).

In the OPE approximation, the nucleons interact with each other exchanging one pion $\pi$. This interaction is described by the effective vertex $\left(2 m f_{i j} / m_{\pi}\right) \bar{N}_{i} \gamma_{5} N_{j} \pi$, where $m$ is the nucleon mass $\left(m_{n} \simeq m_{p}\right)$ and $f_{i j} \sim 1$ is a phenomenological constant $(i, j=n, p)$. It depends on whether the pion is chargeless or not, being $f_{n p}=$ $\sqrt{2} f_{n n}=-\sqrt{2} f_{p p}$, as required by the isospin invariance. Analogously, the axion-nucleon interaction is $\left(g_{i} / 2 m\right) \bar{N}_{i} \gamma_{\mu} \gamma_{5} N_{i} \partial^{\mu} a$, where the axion-nucleon couplings are defined as $g_{n}=c_{n} m / f_{a}$ and $g_{p}=c_{p} m / f_{a} \cdot{ }^{12}$ For the constants $c_{n} \sim c_{p} \sim 1$, they are generally model independent, since the axionnucleon interaction arises mainly from axionpion mixing. In the following, we will consider the general case $g_{n} \neq g_{p}$.

As in the text, we indicate the nucleon momenta with $p_{i} \simeq\left(m+\mathbf{p}_{i}^{2} / 2 m, \mathbf{p}_{\mathbf{i}}\right)$ and that of the axion with $a=\left(\omega_{a}, \mathbf{a}\right)$. Also $\mathbf{k}=\mathbf{p}_{2}-\mathbf{p}_{4}$, is the momentum transfer for the direct diagrams, while $\mathbf{l}=\mathbf{p}_{2}-\mathbf{p}_{3}$
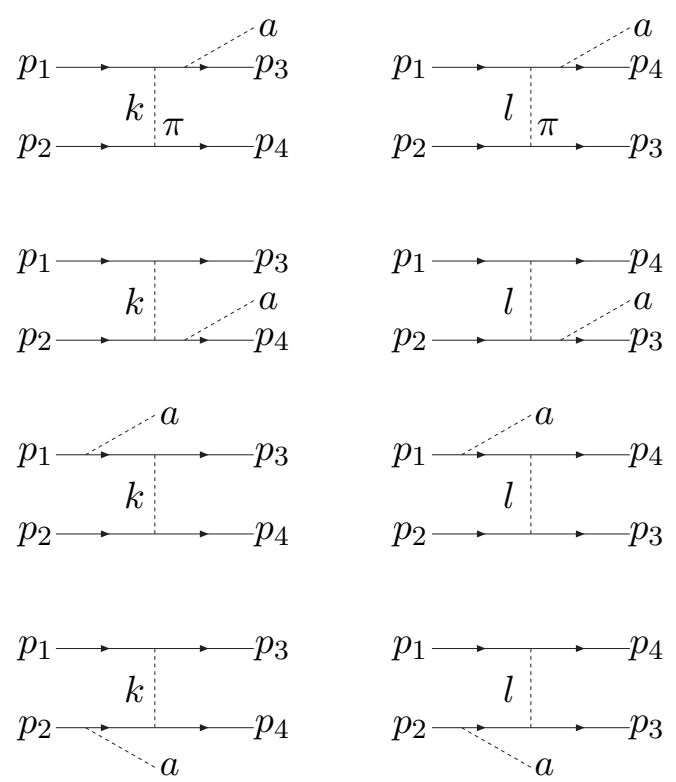

Figure 5: Feynman graphs for $N N \rightarrow N N$ a in the OPE approximation. On the left, from top to bottom, the direct diagrams $A, B, C$, $D$. On the right, from top to bottom, the exchange diagrams $A^{\prime}, B^{\prime}, C^{\prime}, D^{\prime}$. refers to the exchange diagrams.

There are 8 different Feynman graphs that contribute to the process under examination: 4 direct $(A, B, C, D)$ and for 4 exchange $\left(A^{\prime}, B^{\prime}, C^{\prime}, D^{\prime}\right)$ diagrams. ${ }^{13}$ The total matrix element squared is then given by $\mathcal{M}^{2}=\left(A+B+C+D+A^{\prime}+B^{\prime}+C^{\prime}+D^{\prime}\right)^{2}$. The different contributions have the form:

$$
\begin{aligned}
& X=\frac{1}{|\mathbf{k}|^{2}+m_{\pi}^{2}} \frac{1}{ \pm 2 p_{i} \cdot a+m_{a}^{2}} \frac{2 m}{m_{\pi}^{2}} \Omega_{X}, \\
& X^{\prime}=\frac{1}{|\mathbf{l}|^{2}+m_{\pi}^{2}} \frac{1}{ \pm 2 p_{i} \cdot a+m_{a}^{2}} \frac{2 m}{m_{\pi}^{2}} \Omega_{X^{\prime}},
\end{aligned}
$$

where $X$ and $X^{\prime}$ indicate respectively $A, B, C, D$ and $A^{\prime}, B^{\prime}, C^{\prime}, D^{\prime}$, and the index $i$ refers to the nucleons at which the axion leg is attached. The "+" sign, in the denominator, applies to the diagrams $A, B, A^{\prime}, B^{\prime}$, and the "-" to $C, D, C^{\prime}, D^{\prime}$.

\footnotetext{
${ }^{12}$ In general, the nucleon-pion interaction has the derivative form $\left(f_{i j} / m_{\pi}\right) \bar{N}_{i} \gamma_{\mu} \gamma_{5} N_{j} \partial^{\mu} \pi$, typical of the (pseudo-) Goldstone modes, just as the axion. However, this interaction can be made pseudoscalar (as in the main text), after an opportune chiral rotation of the nucleon fields. Yet, this operation cannot be performed for both the pion and the axion field at once. See ref. 17 in [15] for more details

${ }^{13}$ The diagrams are the same, and have the same name, as in reference [15]. We use the same notation here for convenience.
} 
For the general nucleon-nucleon axion bremsstrahlung process, $\alpha \beta \rightarrow \alpha \beta$ a, the functions $\Omega_{X}, \Omega_{X^{\prime}}$ are expressed as:

$$
\begin{aligned}
& \Omega_{A, C}=f_{\alpha \alpha} f_{\beta \beta}{\overline{u_{3}}}^{(\alpha)} \Gamma_{A, C} u_{1}^{(\alpha)} \bar{u}_{4}^{(\beta)} \gamma_{5} u_{2}^{(\beta)} g_{\alpha} \\
& \Omega_{A^{\prime}, C^{\prime}}=f_{\alpha \beta}^{2}{\overline{u_{4}}}^{(\beta)} \Gamma_{A^{\prime}, C^{\prime}} u_{1}^{(\alpha)} \bar{u}_{3}^{(\alpha)} \gamma_{5} u_{2}^{(\beta)} \times \begin{cases}g_{\beta} & \left(\text { for } A^{\prime}\right) \\
g_{\alpha} & \left(\text { for } C^{\prime}\right)\end{cases} \\
& \Omega_{B, D, B^{\prime}, D^{\prime}}=\Omega_{A, C, A^{\prime}, C^{\prime}}(1 \leftrightarrow 2,3 \leftrightarrow 4, \alpha \leftrightarrow \beta),
\end{aligned}
$$

where the notation $u_{i}$ is a short for $u\left(p_{i}\right)$, and the indexes $\alpha$ and $\beta$ stand for the neutron $(n)$ or the proton $(p)$. For example, in the $n p$ bremsstrahlung the two spinors $u^{(\alpha)}, u^{(\beta)}$ represent respectively the neutron and proton field and $g_{a}=g_{n}, g_{b}=g_{p}$, while for the $n n$ or $p p$ bremsstrahlung, $u^{(\alpha)} \equiv u^{(\beta)}$ and $g_{\alpha}=g_{\beta}$. Finally, the matrix functions $\Gamma$ are

$$
\begin{array}{ll}
\left.\Gamma_{A}=m_{a}^{2}+\not d(\not)_{3}-m\right), & \left.\Gamma_{C}=m_{a}^{2}-(\not)_{1}-m\right) d, \\
\Gamma_{B, D}=\Gamma_{A, C}(1 \leftrightarrow 2,3 \leftrightarrow 4), & \Gamma_{X^{\prime}}=\Gamma_{X}(3 \leftrightarrow 4) .
\end{array}
$$

We have computed the matrix element squared, summed over the nucleon spin, in the hypotheses $(i)$ and $(i i)$ of section 3 . As explained, these imply that the axion threemomentum is negligible with respect to that typical of the nucleons.

An important consequence of $(i)$ and $(i i)$ is that $\mathbf{p}_{i} \cdot \mathbf{a} \leq\left|\mathbf{p}_{i}\right||\mathbf{a}| \ll m \omega_{a}$, and $m_{a}^{2} \ll$ $m \omega_{a}$, so that the second denominator of both equations (16) is simply $\sim \pm m \omega_{a}$. Another consequence is that the axion mass is always negligible with respect to $|\mathbf{k}|^{2}$ and $|\mathbf{l}|^{2} \sim 3 m T$. This considerably simplifies the computation of the matrix element squared.

The matrix $\sum_{\text {spin }}|\mathcal{M}|^{2}$ contains three different contributions: $i$ ) a term from the product of two direct diagrams, which gives a contribution proportional to $\left.\left(|\mathbf{k}|^{2}+m_{\pi}^{2}\right)^{-2} ; i i\right)$ a term from the product of two exchange diagrams, which gives a contribution proportional to $\left(|\mathbf{l}|^{2}+\right.$ $\left.m_{\pi}^{2}\right)^{-2}$; and finally, iii) a term from the product of one direct and one exchange diagram, which gives a contribution proportional to $\left(|\mathbf{k}|^{2}+m_{\pi}^{2}\right)^{-1}\left(|\mathbf{l}|^{2}+m_{\pi}^{2}\right)^{-1}$. A straightforward, though very long, calculation leads to:

$$
\sum_{\text {spin }}|\mathcal{M}|^{2}=\frac{32}{9} \frac{m^{2}}{m_{\pi}^{4}}\left(\frac{\mathbf{a}^{2}}{\omega_{a}^{2}}\right)\left\{\frac{C_{k}|\mathbf{k}|^{4}}{\left(|\mathbf{k}|^{2}+m_{\pi}^{2}\right)^{2}}+\frac{C_{l}|\mathbf{l}|^{4}}{\left(|\mathbf{l}|^{2}+m_{\pi}^{2}\right)^{2}}+\frac{C_{k l}|\mathbf{k}|^{2}|\mathbf{l}|^{2}-3 C_{k \cdot l}|\mathbf{k} \cdot \mathbf{l}|^{2}}{\left(|\mathbf{k}|^{2}+m_{\pi}^{2}\right)\left(|\mathbf{l}|^{2}+m_{\pi}^{2}\right)}\right\},
$$

where we have averaged over the axion emission angles: $\left\langle(\hat{\mathbf{k}} \cdot \hat{\mathbf{a}})^{2}\right\rangle=\frac{1}{3},\left\langle(\hat{\mathbf{l}} \cdot \hat{\mathbf{a}})^{2}\right\rangle=\frac{1}{3}$. Observe that the dependence of (19) on the axion three-momentum, satisfies the requirement $|\mathcal{M}|^{2} \rightarrow 0$ for $\mathbf{a} \rightarrow 0$, deducible from general considerations on the (pseudo-)Goldstone nature of the axion, as discussed in section 3 .

For the coefficients in (19) we found, in general:

$$
\begin{aligned}
C_{k} & =12 f^{4}\left(g_{\alpha}^{2}+g_{\beta}^{2}\right), & C_{l} & =3 f_{\alpha \beta}^{4}\left(3 g_{\alpha}^{2}+3 g_{\beta}^{2}+2 g_{\alpha} g_{\beta}\right), \\
C_{k l} & =12 f^{2} f_{\alpha \beta}^{2}\left(g_{\alpha}^{2}+g_{\beta}^{2}\right), & C_{k \cdot l} & =8 f^{2} f_{\alpha \beta}^{2}\left(g_{\alpha}^{2}+g_{\beta}^{2}+g_{\alpha} g_{\beta}\right),
\end{aligned}
$$

where $f=f_{n n}=-f_{p p} \cdot{ }^{14}$

\footnotetext{
${ }^{14}$ Observe that there is a relative minus sign between direct and exchange diagrams for the case of $n n$ or $p p$ process. This is taken into account in the definition of the parameters (20). In fact, in $C_{k l}$ and $C_{k \cdot l}$, $f_{\alpha \alpha} f_{\beta \beta}$ should appear in place of $f^{2}$. So, for the $n p$ process, $C_{k l}$ and $C_{k \cdot l}$ are written in (20) with the wrong sign. However, this is compensated by the sign of $C_{k l}$ and $C_{k \cdot l}$ in (19), which should be the opposite for the n $p$ bremsstrahlung.
} 
In the simple cases of $n n$ or $p p$ processes, relations (20) lead to the simple result:

$$
C_{k}=C_{l}=C_{k l}=C_{k \cdot l}=24 f^{4} g_{n}^{2},
$$

that agrees with eq (6) (observe that in this case $S=1 / 4$, and remember that $\alpha_{\pi}=$ $\left.\left(2 \mathrm{fm} / \mathrm{m}_{\pi}\right)^{2} / 4 \pi\right)$. The more complicated $n p$ scattering, instead, requires:

$$
\begin{aligned}
& C_{k}=12 f^{4}\left(g_{n}^{2}+g_{p}^{2}\right), \quad C_{l}=12 f^{4}\left(3 g_{n}^{2}+3 g_{p}^{2}+2 g_{n} g_{p}\right), \\
& C_{k l}=24 f^{4}\left(g_{n}^{2}+g_{p}^{2}\right), \quad C_{k \cdot l}=16 f^{4}\left(g_{n}^{2}+g_{p}^{2}+g_{n} g_{p}\right),
\end{aligned}
$$

which leads to the matrix element squared:

$$
\begin{aligned}
\sum_{\operatorname{spin}}|\mathcal{M}|^{2}=\frac{128}{3} \frac{m^{2} f^{4}}{m_{\pi}^{4}}\left(\frac{\mathbf{a}^{2}}{\omega_{a}^{2}}\right) & \left\{\frac{\left(g_{n}^{2}+g_{p}^{2}\right)|\mathbf{k}|^{4}}{\left(|\mathbf{k}|^{2}+m_{\pi}^{2}\right)^{2}}+\frac{\left(3 g_{n}^{2}+3 g_{p}^{2}+2 g_{n} g_{p}\right)|\mathbf{l}|^{4}}{\left(|\mathbf{l}|^{2}+m_{\pi}^{2}\right)^{2}}\right. \\
+ & \left.\frac{2\left(g_{n}^{2}+g_{p}^{2}\right)|\mathbf{k}|^{2}|\mathbf{l}|^{2}-\frac{4}{3}\left(g_{n}^{2}+g_{p}^{2}+g_{n} g_{p}\right)|\mathbf{k} \cdot \mathbf{l}|^{2}}{\left(|\mathbf{k}|^{2}+m_{\pi}^{2}\right)\left(|\mathbf{l}|^{2}+m_{\pi}^{2}\right)}\right\}
\end{aligned}
$$

In the limit $m_{a}=0$ this corresponds to the result in ref. [15].

In what follows, we will refer to the $n n$ process (the $p p$ process is equivalent), unless we specify otherwise.

For the computation of the axion emission rate, it is rather convenient to introduce the new set of variables: ${ }^{15}$ the center of mass momenta $\mathbf{p}_{1,2}=\mathbf{P} \pm \mathbf{p}_{i}, \mathbf{p}_{3,4}=\mathbf{P}^{\prime} \pm \mathbf{p}_{f}$, where $\mathbf{P}=\frac{1}{2}\left(\mathbf{p}_{1}+\mathbf{p}_{2}\right)$ and $\mathbf{P}^{\prime}=\frac{1}{2}\left(\mathbf{p}_{3}+\mathbf{p}_{4}\right)$; the cosine of the nucleon scattering angle $z=\hat{\mathbf{p}}_{i} \cdot \hat{\mathbf{p}}_{f} ;$ the adimensional parameters: $u=\mathbf{p}_{i}^{2} / m T, v=\mathbf{p}_{f}^{2} / m T, y=m_{\pi}^{2} / m T, x=\omega / T$, and $q=m_{a} / T$. Thus the matrix element squared can be conveniently expressed as:

$$
\left\{\mathcal{M}^{2}\right\}_{n n}=\zeta^{2} \frac{64 \pi^{2} \alpha_{\pi}^{2}}{3 m^{2}} g_{n}^{2} \eta
$$

where $\zeta=\left(1-q^{2} / x^{2}\right)^{1 / 2}$ is the axion velocity, and $\eta=\left(\eta_{\mathbf{k}}+\eta_{\mathbf{l}}+\eta_{\mathbf{k} \mathbf{l}}-3 \eta_{\mathbf{k} \cdot \mathbf{l}}\right)$,

$$
\begin{array}{ll}
\eta_{\mathbf{k}}=\left(\frac{u+v-2 z \sqrt{u v}}{u+v-2 z \sqrt{u v}+y}\right)^{2}, & \eta_{\mathbf{l}}=\left(\frac{u+v+2 z \sqrt{u v}}{u+v+2 z \sqrt{u v}+y}\right)^{2}, \\
\eta_{\mathbf{k} \mathbf{l}}=\frac{(u+v)^{2}-4 u v z}{(u+v+y)^{2}-4 u v z}, & \eta_{\mathbf{k} \cdot \mathbf{l}}=\frac{(u-v)^{2}}{(u+v+y)^{2}-4 u v z}
\end{array}
$$

Observe that the axion velocity $\zeta$ measures the only contribution of finite axion mass to the matrix element squared.

For negligible axion three-momentum (with respect to that of the nucleons) the delta function in (3) is simply $\delta^{3}\left(\mathbf{P}-\mathbf{P}^{\prime}\right) \delta(u-v-x) / T$. Moreover the axion distribution is isotropic, since we have already averaged over the axion momentum directions. Hence, $d \Pi_{a}=(T / 2 \pi)^{2} \zeta x d x$, and expression (3) can be recasted in the more convenient form:

$$
T \frac{d \mathcal{N}}{d x}=\frac{35}{128} \tilde{Q} \zeta^{3} x \int d u \int d v \sqrt{u v} e^{-u} \delta(u-v-x) \frac{1}{2} \int_{-1}^{1} \eta d z,
$$

\footnotetext{
${ }^{15}$ The notation here strictly follows the appendix of ref. [16].
} 
where the constant factor

$$
\tilde{Q}=\frac{32}{105} \frac{\rho^{2} T^{7 / 2} \alpha_{\pi}^{2}}{\pi^{3 / 2} m^{13 / 2}} g_{n}^{2} \simeq 3.4 \times 10^{42} T_{\mathrm{MeV}}^{7 / 2} \rho_{12}^{2} g_{n}^{2} \operatorname{erg~cm}^{-3} \mathrm{~s}^{-1}
$$

is related to the axion energy emission rate for $m_{a}=m_{\pi}=0$ (8), as we are going to show. In fact we can write the axion energy emission rate as:

$$
Q=T \int_{q}^{\infty} x \frac{d \mathcal{N}}{d x} d x
$$

Observe that this corresponds to relation (B-6) in ref.[16], except for the correction factor $\zeta^{3}$ in (26) and the lower integration limit $q$. In the limit of negligible pion mass, $\eta$ reduces to $\eta_{0}=3\left(1-\eta_{\mathbf{k} \cdot \mathbf{l}}\right)$. We see that, for $m_{a}=0$, the first term in $\eta_{0}$ contributes to $Q$ as $3 \tilde{Q}$. If we define $Q_{\mathbf{k} \cdot \mathbf{l}}$ as the contribution to $Q$ from $3 \eta_{\mathbf{k} \cdot \mathbf{l}}$, then, (still in the limit $m_{a}=0$ ), the second term in $\eta_{0}$ contributes to $Q$ as $-\beta \tilde{Q}$, where $\beta=Q_{\mathbf{k} \cdot \mathbf{l}} / \tilde{Q} \simeq 1.31$. The same argument can be repeated for $\mathcal{N}$, defining $3 \tilde{\mathcal{N}}$ as the contribution to $\mathcal{N}$ from the first term in $\eta_{0}, \mathcal{N}_{\mathbf{k} \cdot \mathbf{l}}$ as the contribution form the second term in $\eta_{0}$, and $\beta^{\prime}=\mathcal{N}_{\mathbf{k} \cdot \mathbf{l}} / \tilde{\mathcal{N}} \simeq 1.02$. Finally, we recover (8) and (9) in the form: ${ }^{16}$

$$
Q_{0}=\tilde{Q}(3-\beta), \quad \mathcal{N}_{0}=\tilde{\mathcal{N}}\left(3-\beta^{\prime}\right)
$$

Notice that the direct substitution of $|\hat{\mathbf{k}} \cdot \hat{\mathbf{l}}|^{2}$ with $\beta / 3$ in the matrix (19) (see, e.g., 15]) can be incorrect, even in the limit $m_{a}=0, y \ll 1$, and is strictly valid only concerning the contribution to the axion energy emission rate. For example, the substitution above would have brought the result $\mathcal{N}_{0}=\tilde{\mathcal{N}}(3-\beta) \simeq 1.69 \tilde{\mathcal{N}}$, instead of $\mathcal{N}_{0}=\tilde{\mathcal{N}}\left(3-\beta^{\prime}\right) \simeq 1.98 \tilde{\mathcal{N}}$. However the error that results is less than $20 \%$, which is usually negligible with respect to other approximations necessary for the calculation (see, e.g., the discussion in [17, page $120)$.

For the sake of comparison with other papers, we consider the dynamical contribution to the axion energy emission rate $Q$, in the limit of zero pion mass, in the $n p$ bremsstrahlung. Suppose $g_{n}=g_{p}=g_{N}$. Then, from (23), and substituting $|\hat{\mathbf{k}} \cdot \hat{\mathbf{l}}|^{2}=\beta / 3$, we find

$$
\left\{\mathcal{M}^{2}\right\}_{n p}=\zeta^{2} \frac{256}{3} \frac{m^{2} f^{4}}{m_{\pi}^{4}}(7-2 \beta)
$$

that means $\left\{\mathcal{M}^{2}\right\}_{n p}=4[(7-2 \beta) /(3-\beta)]\left\{\mathcal{M}^{2}\right\}_{n n}$, about 10.4 times larger. This agrees with the results in [15, 18, in the limit of zero axion mass. The analogous contribution to the axion emission rate $\mathcal{N}$, has the same expression as above, but with $\beta \rightarrow \beta^{\prime}$, and leads to $\mathcal{N} \sim 10$ times larger. The average energy of the emitted axions in the $n p$ bremsstrahlung, is then slightly larger, $\bar{\omega}_{a} \sim 2.02$, with respect to the $n n$ or $p p$ process.

We finally consider the mean free path. In this case the relevant process is the axion absorption by the nuclear medium $N N a \rightarrow N N$. Thus the axion energy appears in the $\delta$ function with the opposite sign with respect to (26). The axion mean free path can then be written as

$$
\lambda^{-1}=\frac{1}{2 \zeta x T} \frac{d \mathcal{N}(-x)}{d \Pi_{a}}=\frac{2 \pi^{2}}{\zeta^{2} x^{2} T^{3}} \frac{d \mathcal{N}(-x)}{d x} .
$$

\footnotetext{
${ }^{16}$ It is useful to notice that $\int d u d v d x x^{n} \sqrt{u v} e^{-u} \delta(u-v-x)=8 / 5$ for $n=1$, and $128 / 35$ for $n=2$. Thus $\tilde{\mathcal{N}}=(7 / 16) \tilde{Q} / T$.
} 
Eliminating the integration over $v$, by virtue of the $\delta$ function, we get

$$
\lambda^{-1}=\frac{35 \pi^{2}}{64} \frac{\tilde{Q}}{T^{4}} \zeta x^{-1} f(x, y)
$$

where

$$
f(x, y)=\int_{0}^{\infty} d u((u+x) u)^{1 / 2} e^{-u} \frac{1}{2} \int_{-1}^{1} \eta d z .
$$

Observe that, if $\eta$ is constant, $f(x, y)$ can be analytically expressed in terms of the modified Bessel functions. In ref. [18, it is assumed $\eta \simeq 3-\beta$, which is a pretty good approximation in the limit $y \ll 1$ (see the discussion above). In this case, expression (33) reduces to

$$
\frac{1}{2}(3-\beta) x e^{x / 2} K_{1}(x / 2),
$$

and, consequently,

$$
\lambda^{-1} \simeq \frac{35 \pi^{2}}{128} \frac{Q_{0}}{T^{4}} \zeta e^{x / 2} K_{1}(x / 2)=2.5 \times 10^{6} T_{\mathrm{MeV}}^{-1 / 2} \rho_{12}^{2} g_{n}^{2} \zeta e^{x / 2} K_{1}(x / 2) \mathrm{cm}^{-1},
$$

where we have used $\tilde{Q}(3-\beta)=Q_{0}$. This expression leads directly to the results (13) and (15) in section 3 .

\section{References}

[1] R. D. Peccei and H. R. Quinn, Phys. Rev. D 16, 1791 (1977).

[2] For a general review on axion physics see, e.g., J. E. Kim, Phys. Rept. 150 (1987) 1; H.Y. Cheng, ibid. 158 (1988) 1. The axion phenomenology, in particular in relation with the astrophysical processes, is largely discussed also in G. G. Raffelt, Phys. Rept. 198 (1990) 1; and in M. S. Turner, Phys. Rept. 197 (1990) 67. A short account of recent results can be found in G. G. Raffelt, arXiv: hep-ph/0504152. A more general discussion about axions and other weakly interacting particles can be found in ref. 17, and in G. G. Raffelt, Part. Sci. 49 (1999) 163 [arXiv:hep-ph/9903472].

[3] S. Weinberg, Phys. Rev. Lett. 40 (1978) 223; F. Wilczek, ibid. 40, 279 (1978).

[4] A.R. Zhitnitskii, Sov. J. Nucl. Phys. 31 (1980) 260; M. Dine, W. Fischler and M. Srednicki, Phys. Lett. B 104 (1981) 199.

[5] J.E. Kim, Phys. Rev. Lett. 43 (1979) 103; M. A. Shifman, A. I. Vainshtein and V. I. Zakharov, Nucl. Phys. B 166 (1980) 493.

[6] Z. Berezhiani, Phys. Lett. B 129 (1983) 99; Phys. Lett. B 150 (1985) 177;

A. Anselm, Z. Berezhiani, Phys. Lett. B 162 (1985) 349;

Z. Berezhiani, M. Khlopov, Z. Phys. C 49 (1991) 73; Sov. J. Nucl. Phys. 51 (1990) 739 ; 51 (1990) 935;

Z.G. Berezhiani, M.Yu. Khlopov, R.R. Khomeriki, Sov. J. Nucl. Phys. 52 (1990) 65; ibid. 52, 344 (1990).

[7] G. R. Dvali, arXiv:hep-ph/9505253

M. Giannotti, to be published in Int. J. Mod. Phys. A, [arXiv:astro-ph/0504636]. 
[8] Z. Berezhiani, L. Gianfagna, M. Giannotti, Phys. Lett. B 500 (2001) 286; The idea that the interaction with a hidden sector of particles would change the usual relation between axion mass and PQ constant was originally proposed in V.A. Rubakov, JETP Lett. 65 (1997) 621.

[9] L. Gianfagna, M. Giannotti and F. Nesti, JHEP $0410 \quad$ (2004) 044 [arXiv:hep-ph/0409185] .

[10] Z. Berezhiani and A. Drago, Phys. Lett. B 473, 281 (2000).

[11] The nucleon-nucleon axion bremsstrahlung process was first described in the degenerate limit in N. Iwamoto, Phys. Rev. Lett. 53 (1984) 1198. The non-degenerate case was considered in M.S. Turner, Phys. Rev. Lett. 60 (1988) 1797, and later in other papers (see, e.g., 15, 16]). An accurate discussion of the emission for any degeneracy is given in [15].

[12] P. Meszaros and M.J. Rees, Astrophys. J. 476 (1997) 261;

M. Vietri, Astrophys. J. 488 (1997) L105;

E. Waxmann, Astrophys. J. 485 (1997) L5.

[13] M. Ruffert and H.-Th. Janka, arxiv:astro-ph/9804132

[14] M. S. Turner, H. S. Kang and G. Steigman, Phys. Rev. D 40 (1989) 299.

[15] R. P. Brinkmann, M.S. Turner, Phys. Rev. D 38 (1988) 3297.

[16] G. Raffelt and D. Seckel, Phys. Rev. D 52 (1995) 1780.

[17] G.G. Raffelt, Stars as Laboratories for Fundamental Physics, the university of Chicago press (Chicago \& London).

[18] A. Burrows, M.T. Ressell, M.S. Turner, Phys. Rev. D 42 (1990) 3297. 